\title{
The role of indocyanine green videoangiography with FLOW 800 analysis for the surgical management of central nervous system tumors: an update
}

\author{
Francesco Acerbi, MD, PhD, Ignazio G. Vetrano, MD, Tommaso Sattin, MD, \\ Camilla de Laurentis, MD, Lorenzo Bosio, MD, Zefferino Rossini, MD, Morgan Broggi, MD, PhD, \\ Marco Schiariti, MD, and Paolo Ferroli, MD
}

Neurosurgical Unit II, Department of Neurosurgery, Fondazione IRCCS “Istituto Neurologico Carlo Besta," Milan, Italy

OBJECTIVE Indocyanine green videoangiography (ICG-VA) is an intraoperative technique used to highlight vessels in neurovascular surgery. Its application in the study of the vascular pathophysiology in CNS tumors and its role in their surgical management are still rather limited. A recent innovation of ICG-VA (i.e., the FLOW 800 algorithm integrated in the surgical microscope) allows a semiquantitative evaluation of cerebral blood flow. The aim of this study was to evaluate for the first time the systematic application of ICG-VA and FLOW 800 analysis during surgical removal of CNS tumors.

METHODS Between May 2011 and December 2017, all cases in which ICG-VA and FLOW 800 analysis were used at least one time before, during, or after the tumor resection, and in which surgical videos were available, were retrospectively reviewed. Results of the histological analysis were analyzed together with the intraoperative ICG-VA with FLOW 800 in order to investigate the tumor-related videoangiographic features.

RESULTS Seventy-one patients who underwent surgery for cerebral and spinal tumors were intraoperatively analyzed using ICG-VA with FLOW 800, either before or after tumor resection, for a total of 93 videoangiographic studies. The histological diagnosis was meningioma in 25 cases, glioma in 14, metastasis in 7, pineal region tumor in 5, hemangioblastoma in 4, chordoma in 3, and other histological types in 13 cases. The authors identified 4 possible applications of ICG-VA and FLOW 800 in CNS tumor surgery: extradural surveys allowed exploration of sinus patency and the course of veins before dural opening; preresection surveys helped in identifying pathological vascularization (arteriovenous fistulas and neo-angiogenesis) and regional venous outflow, and in performing temporary venous clipping tests, when necessary; postresection surveys were conducted to evaluate arterial and venous patency and parenchymal perfusion after tumor removal; and a premyelotomy survey was conducted in intramedullary tumors to highlight the posterior median sulcus.

CONCLUSIONS The authors found ICG-VA with FLOW 800 to be a useful method to monitor blood flow in the exposed vessels and parenchyma during microsurgical removal of CNS tumors in selected cases. In particular, a preresection survey provides useful information about pathophysiological changes of brain vasculature related to the tumor and aids in the individuation of helpful landmarks for the surgical approach, and the postresection survey helps to prevent potential complications associated with the resection (such as local hypoperfusion or venous infarction).

https://thejns.org/doi/abs/10.3171/2018.3.FOCUS1862

KEYWORDS CNS tumors; FLOW 800; ICG; indocyanine green; software analysis; videoangiography

$\mathrm{M}$ ICROSCOPE-INTEGRATED indocyanine green videoangiography (ICG-VA) has been introduced in vascular neurosurgery in order to visualize cerebral vessels during surgical management of different vascular pathologies. $3,8,9,12,14-19,23,27,28,30-32$ Furthermore,
ICG-VA has been proven to be a useful adjunct for intraoperative study of venous flow dynamics, in both pathologi$\mathrm{cal}^{30,33}$ and normal veins, ${ }^{35}$ and for evaluation of the possibility of venous sacrifice during surgical approaches to different pathologies. ${ }^{35,38}$

ABBREVIATIONS AI = arbitrary intensity unit; AVF = arteriovenous fistula; $\mathrm{GBM}=$ glioblastoma; ICA = internal carotid artery; ICG-VA = indocyanine green videoangiography; $\mathrm{MCA}=$ middle cerebral artery; $\mathrm{PCOA}=$ posterior communicating artery; $\mathrm{ROI}=$ region of interest; $S S S=$ superior sagittal sinus.

SUBMITTED February 1, 2018. ACCEPTED March 22, 2018.

INCLUDE WHEN CITING DOI: 10.3171/2018.3.FOCUS1862. 
In 2011, we were the first group to propose the standard use of ICG-VA to study the vascular pathophysiology in CNS tumors and its role in their surgical management. ${ }^{13}$ Since then, very few reports on the application of ICGVA in the context of CNS tumor resection have been published. ${ }^{7,10,20,22,24,27,36,37}$ More recently, a further development of the software integrated in surgical microscopes (FLOW 800 Software Analysis Tool, Pentero and Kinevo microscopes; Carl Zeiss Co.) allowed investigators to perform a postprocessing semiquantitative analysis of blood flow in arteries, brain parenchyma, and veins, derived from the classic ICG-VA. ${ }^{3,24}$ In this study, we evaluated for the first time the systematic application of ICG-VA with FLOW 800 analysis in a retrospective series of patients who underwent surgical removal of cerebral and spinal tumors.

\section{Methods}

\section{Patient Population}

In the period between May 2011 and December 2017, 1443 patients underwent surgical removal of a CNS tumor at the Neurosurgical Unit II of the Fondazione IRCCS "Istituto Neurologico Carlo Besta." Of these, all cases in which ICG-VA and FLOW 800 analysis were used at least one time before or after the tumor resection, and whose surgical videos and FLOW 800 analysis were available, were retrospectively reviewed in order to explore the different specific applications of this technique during surgical removal of CNS tumors. All patients gave their informed consent to the use of ICG-VA. The surgical database of the Neurosurgical Unit II has been approved by our ethics committee.

\section{Surgery and Postoperative Management}

All patients received a standard preoperative evaluation, including blood testing and general and neurological clinical assessment. All of them underwent a preoperative MRI session, including standard sequences for brain and spinal tumors. Tumor removal was performed with standard technique based on the lesion's location, including the use of neuronavigation, ultrasound, photodynamic detection (tumors were visualized using a fluorescein-guided technique, with the dose of $5 \mathrm{mg} / \mathrm{kg}$, following the recommendation of the FLUOCERTUM study, approved by our ethics committee in 2016), ${ }^{1,2,4,33}$ and neurophysiological monitoring. Tumors were histologically classified based on the 2007 WHO CNS tumor classification before May 2016, and on the current WHO 2016 revision after May 2016. Results of the histological analysis were analyzed together with the intraoperative ICG-VA findings in order to investigate the tumor-related videoangiographic features.

\section{The ICG-VA With FLOW 800 Analysis}

The ICG-VA was performed as previously described. ${ }^{3,12,14}$ Briefly, ICG was administered intravenously by the anesthesiologist upon the surgeon's request, with a standard dose of $12.5 \mathrm{mg}$ in a single bolus, before dural opening, immediately before or after tumor resection. Multiple injections were performed in selected cases, never exceeding the maximum daily dose of $5 \mathrm{mg} / \mathrm{kg} .{ }^{21}$
The flow analysis was based on the use of FLOW 800 software, which was integrated in the surgical microscope (Pentero; Carl Zeiss Meditec). The algorithm calculated fluorescence intensities in the exposed areas based on the average arbitrary intensity units (AIs) detected by the camera and reconstructed maps of maximal fluorescence intensities and of delay times (time interval until 50\% of maximum fluorescence). Specifically, the maps were shown as gray scale of maximal fluorescence intensities and as color scale, depending on time to half-maximal fluorescence. In addition, the course of fluorescence could be further analyzed in any area of the exposed brain, including parenchyma, by using freely definable regions of interest (ROIs) (Fig. 1).

\section{Results}

\section{Patients and CNS Tumors}

A total of 71 cases have been included in this retrospective analysis. There were 61 intracranial tumors: 12 high-grade gliomas (11 glioblastomas [GBMs] and 1 anaplastic oligodendroglioma), 1 low-grade glioma (grade II oligodendroglioma), 2 central neurocytomas, 1 pleomorphic xanthoastrocytoma, 7 metastases, 25 meningiomas (24 grade I and 1 grade II), 5 tumors of the pineal region (4 grade II and 1 grade III pineal parenchymal tumors of intermediate differentiation), 3 chordomas, 1 craniopharyngioma, 3 hemangioblastomas, 1 giant angioma of the cavernous sinus; and 10 spinal cord lesions: 5 ependymomas, 1 pilocytic astrocytoma, 1 neuroendocrine tumor, 1 enterogenic cyst, 1 schwannoma, and 1 hemangioblastoma (Table 1). In 20 cases, the fluorescein-guided technique was used to better visualize and remove the intrinsic tumors enhancing on preoperative MRI.

\section{Applications of ICG-VA With FLOW 800 in CNS Tumor Resection}

General Considerations

The ICG-VA could be performed in all cases, with optimal visualization of arterial, capillary-parenchymal, and venous phases, even in the 20 cases in which the fluorescein-guided technique was used to remove the tumors. No difficulties of tumor visualization with fluorescein due to the simultaneous use of ICG-VA could be detected. In all patients at least 1 injection was performed either before or after tumor resection, with 17 patients receiving multiple injections ( 3 times in 5 patients, 2 times in the remaining cases), for a total of 93 ICG-VA studies (Table 1). No adverse reactions to ICG injections were observed.

\section{Different Applications of ICG-VA During CNS Tumor Resection}

Extradural Survey Before Dural Opening. The ICGVA was used extradurally in 6 cases ( 4 meningiomas, 1 oligodendroglioma, and 1 metastasis). In particular, ICG-VA was used in 3 cases ( 2 parasagittal meningiomas and 1 oligodendroglioma) to show the course of parasagittal cortical veins before dural opening. In 2 torcular meningiomas, ICG-VA showed both the position and patency of the transverse sinus before dural opening. In one case of bulbar metastasis, the use of ICG-VA demonstrated the possibility of 

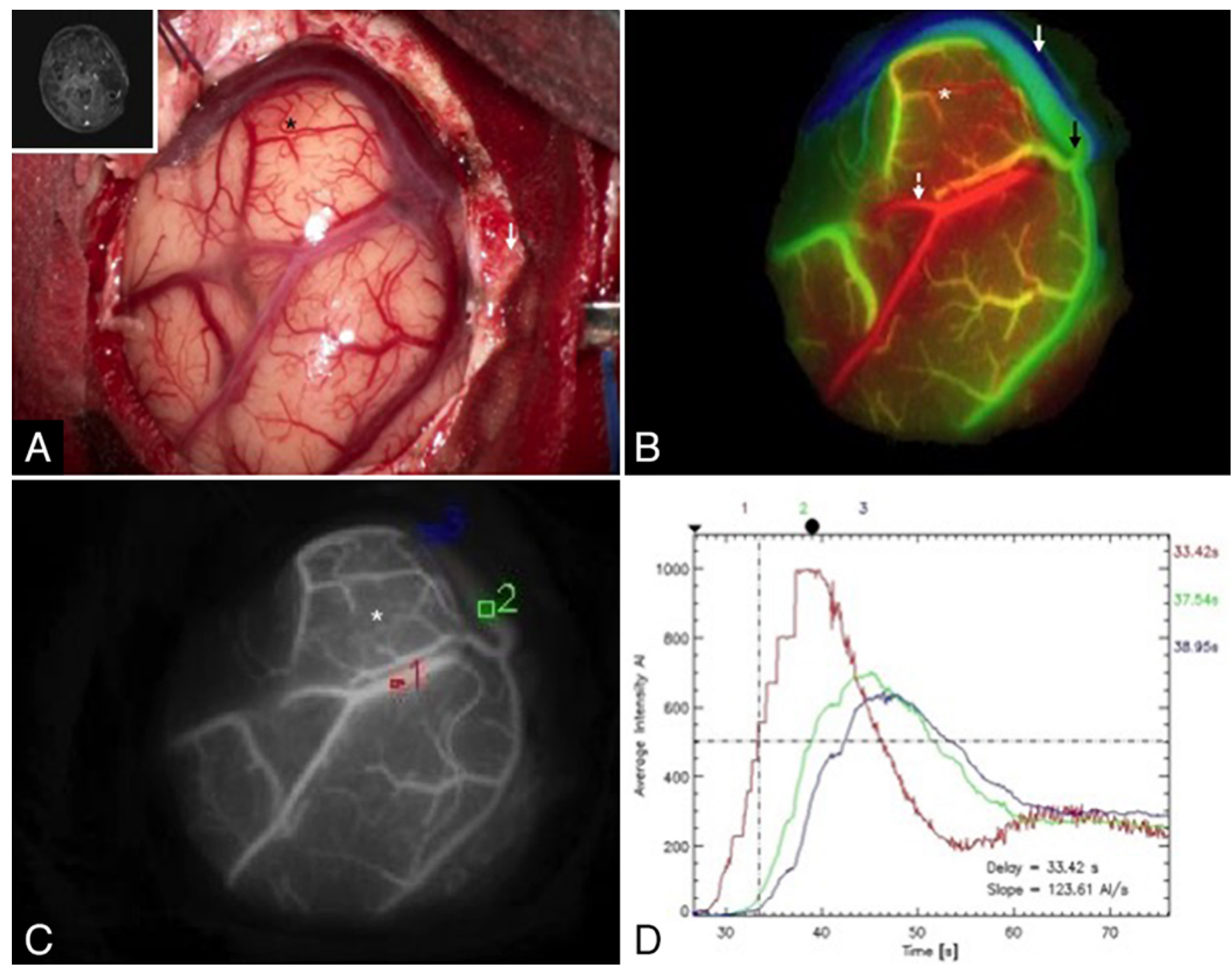

FIG. 1. Example of ICG-VA and FLOW 800 analysis in a case of left temporal metastases from adenocarcinoma (inset: the preoperative MR image). In a white-light microscopy view (A), the superficial projection of the tumor is identified by neuronavigation, as depicted by the asterisk. The ICG FLOW 800 algorithm is able to reconstruct the map of fluorescence intensity (C) based on intensity detected by the camera and graded by the average Als and the map of delay time (B) based on the time to the half-maximal fluorescence in the area exposed by the craniotomy. In this way, arteries are represented in red (dashed arrow), veins in blue (white arrow), and the arterialized portion of the veins in gray/yellow (black arrow). The software also makes it possible to study different ROls in the map of gray intensity, with automatic reconstruction of flow curves over time, as depicted in D. In the graph of this example, the arterial curve is shown in red; the venous curve in blue; and the arterialized part of the vein, with faster flow than normal veins, in green.

sigmoid sinus sacrifice (presence of retrograde flow) for a petro-occipital transsigmoidal surgical route to approach the lesion. We did not find a specific advantage related to the use of FLOW 800 analysis for extradural surveys, due to the fact that all the information could be retrieved with the maps of gray intensity as in classic ICG-VA (Fig. 2).

Preresection Survey. The ICG-VA was performed in 27 cases (37 ICG-VAs) before tumor resection. For parenchymal tumors reaching the brain surface, a superficial vascular survey was used to demonstrate areas of neo-angiogenesis with pathological vessels related to the tumor, or the presence of arteriovenous fistulas (AVFs), with vein flow disturbances in 3 cases of GBM, 3 cases of hemangioblastoma ( 2 cerebellar, 1 cervical), 1 tumor of the pineal region, 1 lumbar intradural neuroendocrine tumor, 1 cervical intradural schwannoma, 1 pleomorphic xanthoastrocytoma, and 1 metastasis (Figs. 3 and 4A-C). In all these cases FLOW 800 analysis allowed investigators to better distinguish the border of the tumors related to neo-angiogenesis and pathological vessels, based on maps of delay time, or the presence of AVFs, both with maps of delay times and analysis of flow curves. In the tumor cases treated with fluorescein-guided removal, $, 2,2,5$ the neoangiogenesis shown on maps of delay times corresponded to the areas highlighted by fluorescein (Fig. 3).

In 4 cases (1 meningioma, 2 GBMs, 1 metastasis) ICGVA showed brain congestion, with a delay of flow particularly in the parenchymal phase due to compression by the 
TABLE 1. Field of application and number of ICG-VA procedures, related to number of cases and tumor histological types

\begin{tabular}{|c|c|c|c|}
\hline Application & $\begin{array}{l}\text { No. of } \\
\text { ICG- } \\
\text { VAs }\end{array}$ & $\begin{array}{l}\text { No. of } \\
\text { Cases }\end{array}$ & Tumor Histological Type \\
\hline \multirow[t]{4}{*}{ Extradural } & 6 & 6 & \\
\hline & & 4 & Meningiomas \\
\hline & & 1 & Oligodendroglioma \\
\hline & & 1 & Metastasis \\
\hline \multirow[t]{12}{*}{ Preresection } & 37 & 27 & \\
\hline & & 7 & GBMs \\
\hline & & 4 & Meningiomas \\
\hline & & 4 & Tumors of the pineal region \\
\hline & & 3 & Hemangioblastomas \\
\hline & & 3 & Metastases \\
\hline & & 1 & Neuroendocrine intradural tumor \\
\hline & & 1 & Cervical intradural schwannoma \\
\hline & & 1 & $\begin{array}{l}\text { Atypical pleomorphic xanthoastro- } \\
\text { cytoma }\end{array}$ \\
\hline & & 1 & Chordoma \\
\hline & & 1 & Spinal ependymoma \\
\hline & & 1 & Intraventricular neurocytoma \\
\hline \multirow[t]{4}{*}{ Myelotomy } & 7 & 7 & \\
\hline & & 5 & Ependymomas \\
\hline & & 1 & Pilocytic astrocytoma \\
\hline & & 1 & Enterogenic cyst \\
\hline \multirow[t]{13}{*}{ Postresection } & 43 & 41 & \\
\hline & & 17 & Meningiomas \\
\hline & & 7 & $\begin{array}{l}\text { HGGs (1 GBM associated w/ PCoA } \\
\text { aneurysm) }\end{array}$ \\
\hline & & 4 & Metastases \\
\hline & & 3 & Chordomas \\
\hline & & 2 & Hemangioblastomas \\
\hline & & 2 & Cervical ependymomas \\
\hline & & 1 & Craniopharyngioma \\
\hline & & 1 & Giant angioma of the cavernous sinus \\
\hline & & 1 & $\begin{array}{l}\text { Atypical meningioma (after decom- } \\
\text { pressive hemicraniectomy in } \\
\text { multiple atypical meningiomas) }\end{array}$ \\
\hline & & 1 & Central neurocytoma \\
\hline & & 1 & Pineal gland tumor \\
\hline & & 1 & $\mathrm{AO}$ \\
\hline
\end{tabular}

$\mathrm{AO}$ = anaplastic oligodendroglioma; $\mathrm{HGG}$ = high-grade glioma.

tumor (1 of the GBMs also presented with vein arterialization) (Fig. 4A-C). The use of FLOW 800 analysis, even if it was not essential to show the brain congestion related to the presence of the tumor, allowed us to identify a slower time to peak due to brain congestion in postprocessing analysis; if repeated postresection, the same analysis could show a steeper flow curve, indicating an improvement of cortical flow (Fig. 4).

In 1 case of recurrent chordoma, a preresection ICGVA session was performed to verify the patency and flow
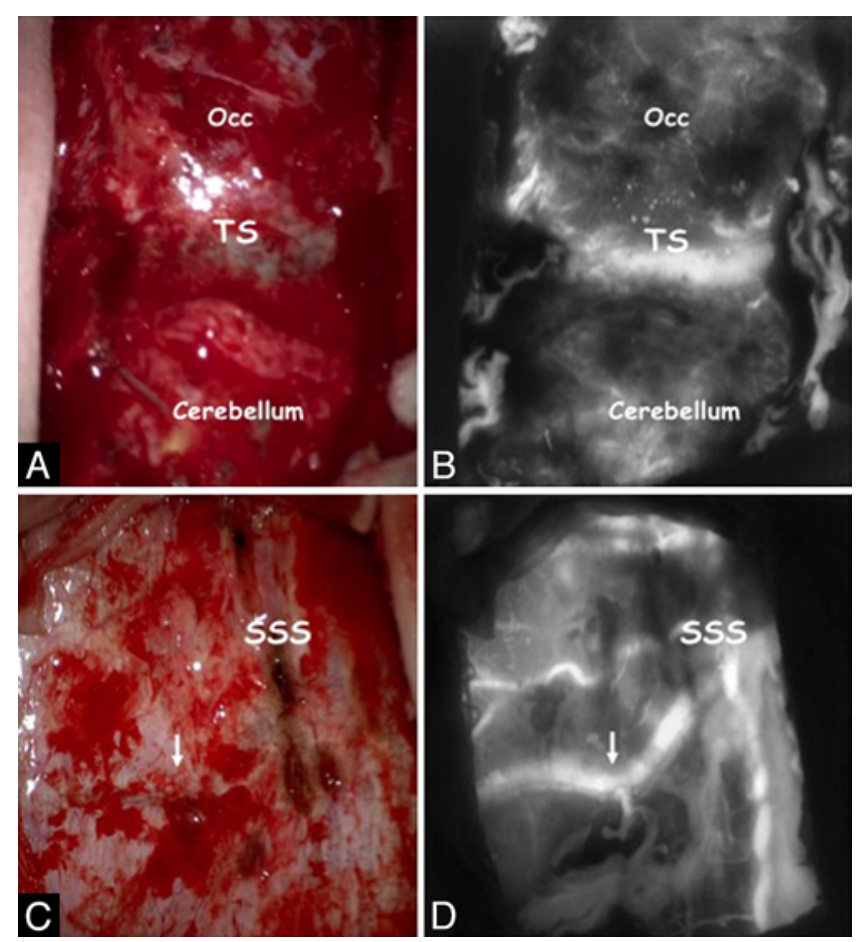

FIG. 2. Extradural survey. A and B: A case of infra- and supratentorial meningioma involving the torcular in which the extradural examination revealed the transverse sinus (TS) between the occipital lobe (Occ) and cerebellar parenchyma. C and D: A case of left frontal oligodendroglioma in which it was possible to identify the SSS and a cortical vein (arrow) draining into the SSS, that was not clearly identifiable in white light (C). The ICG-VA, also with simple maps of gray intensity, guided dural opening, avoiding damage to the sinuses and cortical veins.

characteristics of a vertebral artery that was stenotic due to previous surgery and irradiation. For this last instance, we did not find a specific advantage in the use of FLOW 800 analysis.

In 2 cases ( 1 pineal tumor with 2 injections and 1 parasagittal meningioma) the preresection survey was used to study, with the maps of delay times and flow analysis derived from FLOW 800 software, the specific flow in the veins encountered during the approach (supracerebellar and precerebellar in the case of the pineal tumor), or in the vein completely encased by the tumor (the parasagittal meningioma).

In 9 cases a temporary ICG-VA clipping test was performed to evaluate the possibility of venous sacrifice to either enlarge the surgical corridor to the tumor or completely remove it, as previously described. ${ }^{14,15}$ Briefly, a preclipping ICG-VA study was used to study the basal venous flow, and then it was repeated after temporary clipping of the vein to be potentially sacrificed in order to check for the presence of anastomotic circulation. A total of 18 ICGVAs were therefore performed in this group of patients. In 4 cases (1 intraventricular neurocytoma, 1 frontal metastasis, 1 frontal GBM, 1 meningioma) the test was performed on parasagittal veins draining to the superior sagittal sinus (SSS), that either were obstructing the surgical corridor (the first 3 cases) or were encased by the tumor (the last case of parasagittal meningioma). In 3 cases the postclipping ICG- 

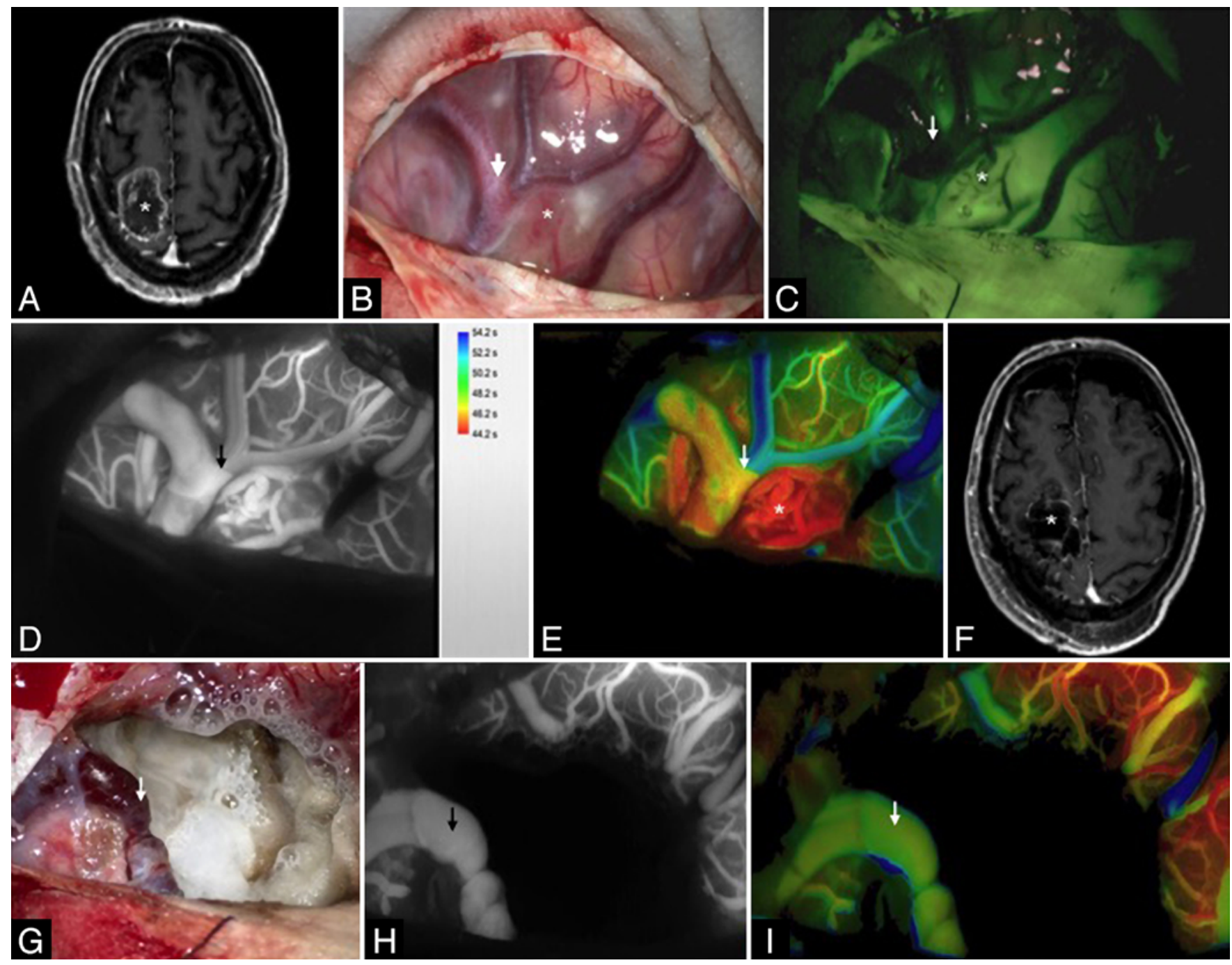

FIG. 3. An example of the use of ICG-VA for a preresection survey in a case of right rolandic GBM treated with fluorescein-guided tumor removal. A: Preoperative T1-weighted MR image obtained with contrast. B: After dural opening, the white-light illumination under microscopic view showed the superficial part of the tumor, which was grayish and with a pathological vessel (asterisk), and an arterialized red flow (white arrow) in the context of a cortical vein draining toward the SSS. C: The superficial part of the tumor was better depicted by activation of the fluorescence module to enhance fluorescein uptake. D: The classic ICG-VA confirmed the point where the fistula and the large cortical vein met (black arrow) and more pathological vessels in the tumoral areas. E: The map of delay time after FLOW 800 analysis better depicted the arterialized flow into the vein (in red-yellow), compared to normal venous flow in blue in the more proximal venous compartment (white arrow); in addition, it could clearly identify the superficial component of the GBM as a red nodule that corresponded to the yellow fluorescence derived from the fluorescein visualization in panel C. G-I: The ICG-VA and FLOW 800 analysis was repeated after tumor removal (G), and showed the flow normalization in the cortical vein and normal perfusion in the parenchyma close to the surgical cavity, both on a map of gray intensity $(H)$ and on one of delay time (I). In F, a postoperative T1-weighted post-Gd MRI scan confirmed total tumor resection.

VA confirmed the presence of anastomotic circulation and the possibility of venous sacrifice. In 1 case the collateral retrograde flow was considered too slow (3 seconds of difference compared to other veins) and the vein was draining an eloquent area (primary motor cortex); thus it was considered unsuitable for a sacrifice. In 3 cases ( 2 tumors of the pineal region and 1 meningioma of the petrous apex) the test was performed on posterior fossa veins. In 1 case the postclipping ICG-VA showed stagnation of superior petrosal veins draining to the superior petrosal sinus (in the petrous apex meningioma), and therefore they were not sacrificed. In 2 cases of pineal region tumors the test was performed on supracerebellar veins, and it was in favor of venous sacrifice, confirming a collateral retrograde flow after temporary clipping. In 1 case (insular GBM) the test was performed on a large sylvian vein connecting temporal and frontal lobe venous systems: the postclipping ICG-VA showed good collateral vessel flow in frontal and temporal systems, and thus the bridging sylvian vein was sacrificed. In 1 case, the clipping test was performed on a vein located in the posterior median sulcus in the spinal cord (in a case of spinal ependymoma). In this case the clipping test was performed directly with bipolar forceps, and it showed no flow perturbation during the temporary 

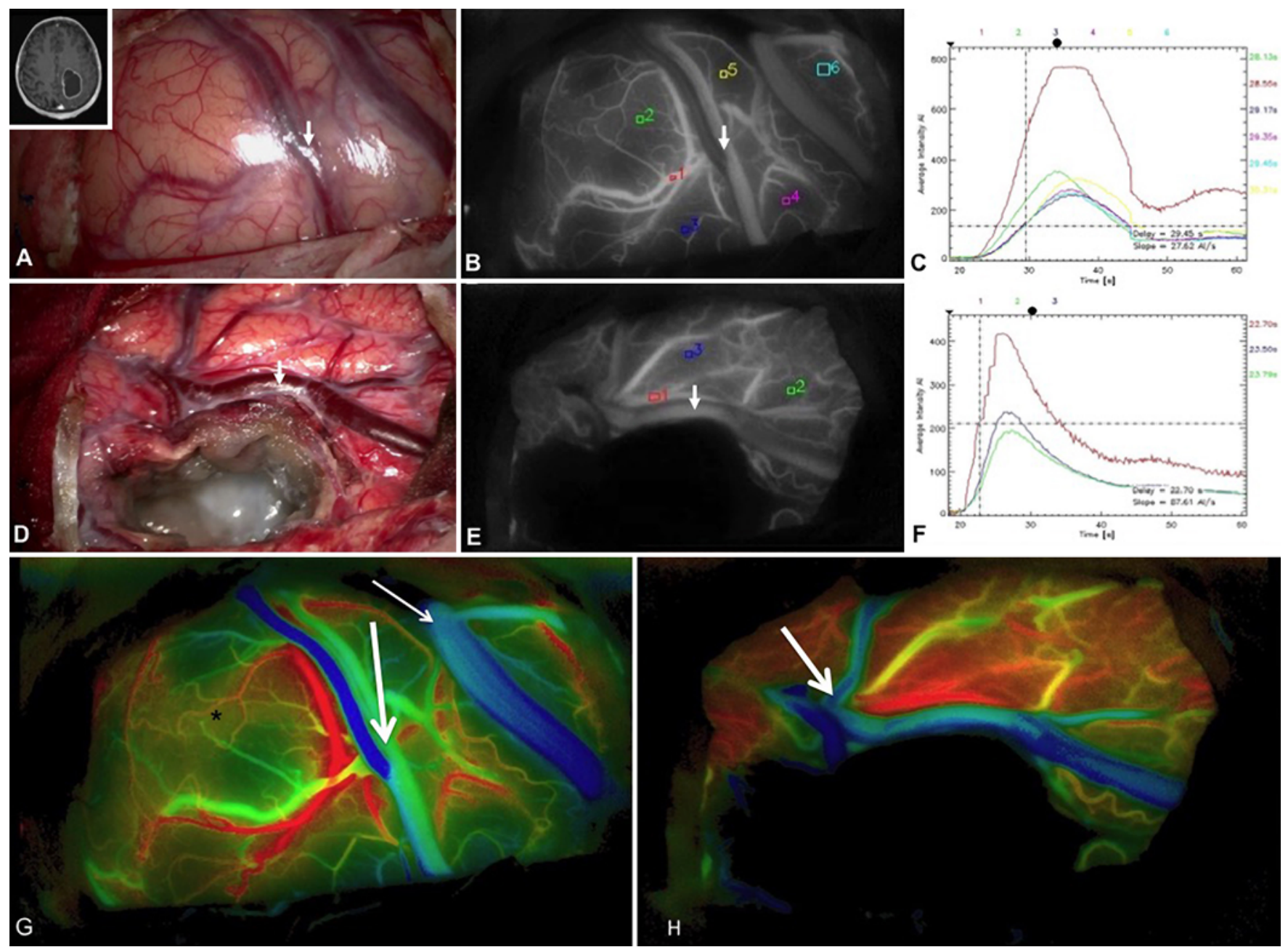

FIG. 4. Preresection and postresection survey in a patient with right parietal GBM. After dural opening the brain appeared swollen and congested. An arterialized flow in a cortical vein was noted (white arrow in $\mathbf{A}$; inset shows preoperative MR image). A preresection survey was performed $(B, C, G)$. The map of delay time $(G)$ allowed us to identify a small area of faster cortical flow (asterisk) in the context of global congestion, and confirmed an AVF in a cortical vein (green vein shown with a large white arrow), and slower and retrograde flow in a more anterior vein related to brain congestion (small white arrow). The flow curve analysis in different ROIs $(B, C)$ showed a low peak for parenchymal curves and slow time to peak, particularly in peripheral regions (curves $5-7)$, due to brain congestion. A postresection survey was completed after tumor resection (D-F, H). The map of delay times $(H)$ showed the normalization of venous flow (large white arrow) and a faster parenchymal flow. The flow curve analysis (E, F) showed a steeper rise time compared to the preresection period, and a slower arterial-capillary difference in time to half-maximal fluorescence, indicating an improvement of brain flow in peritumoral areas.

occlusion; the vein could be therefore sacrificed to enlarge the opening of the posterior median sulcus to completely resect the intramedullary tumor.

The use of FLOW 800 to study venous flow before tumor resection (with or without the clipping test) allowed us to better identify the pattern of venous flow compared to classic ICG-VA, by analyzing the map of delay times and the characteristic flow curves in specific ROIs inside the veins.

Identification of the Posterior Median Sulcus in Spinal Cord Tumors. The ICG-VA was used in 7 cases of spinal cord tumors (5 ependymomas, 1 pilocytic astrocytoma, 1 enterogenic cyst) to better identify the posterior median sulcus to be opened for tumors not abutting the spinal cord surface, based on the position of posterior veins exiting the sulcus (Fig. 5). We did not find a specific advantage for this utilization related to the use of FLOW 800 with the maps of delay time.

Postresection Survey. A postresection ICG-VA was performed in 41 cases (42 ICG-VAs). It was used in 16 cases ( 6 skull base meningiomas, 1 craniopharyngioma, 2 GBMs, 3 metastases, 3 chordomas, 1 giant angioma of the cavernous sinus) to check for patency of arteries directly encased by the tumor. In 12 cases (4 skull base meningiomas, 1 craniopharyngioma, 2 GBMs, 1 metastasis, 3 chordomas, and 1 giant angioma of the cavernous sinus) the ICG-VA confirmed arterial patency with no parenchymal flow disturbances. In 1 case of planum sphenoidalis meningioma infiltrating the optic chiasm and encasing bilaterally the internal carotid artery (ICA) and $\mathrm{A}_{1}$ with their perfo- 


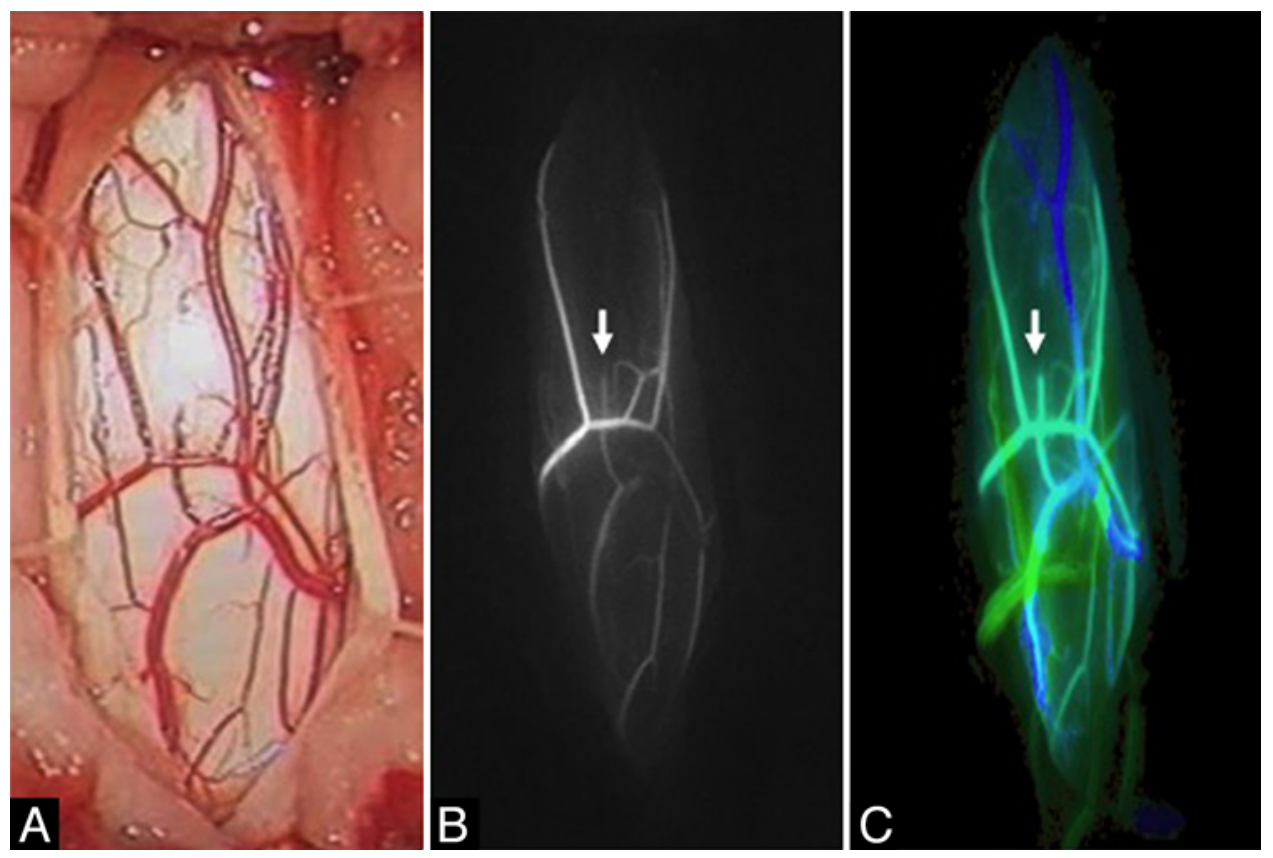

FIG. 5. Intramedullary C2-3 ependymoma not abutting the spinal cord surface: the spinal cord appeared edematous and the median sulcus was not easily distinguishable (A). The ICG-VA study (B) identified a posterior vein (arrow) exiting the median sulcus, improving its recognition. The map of delay times is shown in a corresponding image (C).

rators, postresection ICG-VA with FLOW 800 analysis showed hypoperfusion in the optic chiasm, whereas ICA, $\mathrm{A}_{1}$, and brain cortex were normally perfused in the right hemisphere. The patient suffered severe vision problems due to optic chiasm damage, but no other neurological disturbances were evident in the postoperative period (Fig. 6). In 1 case of right clinoid meningioma with encasement of ICA and middle cerebral artery (MCA), the MCA was damaged during tumor resection and had to be sacrificed. An emergency superficial temporal artery-MCA anastomosis was performed. The ICG-VA with FLOW 800 analysis showed bypass patency with no hypoperfusion in frontotemporal areas exposed by craniotomy. In 1 case of temporal metastasis, an artery completely encased by the tumor was sacrificed without flow perturbation. In 1 sylvian metastatic lesion, an $\mathrm{M}_{4}$ branch was sacrificed and this resulted in hypoperfusion in primary motor cortex, as shown by the map of delay time and the evaluation of flow curves; the hypoperfusion was corrected by the use of a local intracranial-intracranial bypass. ${ }^{3}$ Although the evaluation of arterial patency could be performed also by using the classic ICG-VA, the effect on brain perfusion could be better studied by the use of FLOW 800 analysis.

In 1 case (left temporal GBM), an unruptured posterior communicating artery (PCoA) aneurysm was incidentally discovered during tumor resection and it was clipped in the same surgical session. The ICG-VA was used to check for patency of PCoA and choroidal arteries after clipping, without a specific advantage related to FLOW 800 analysis. ${ }^{5}$

In 10 cases ( 1 insular GBM, 2 cervical ependymomas, 5 parasagittal meningiomas, 1 hemangioblastoma, and 1 decompressive hemicraniectomy for edema and raised intracranial pressure after resection of multiple atypical
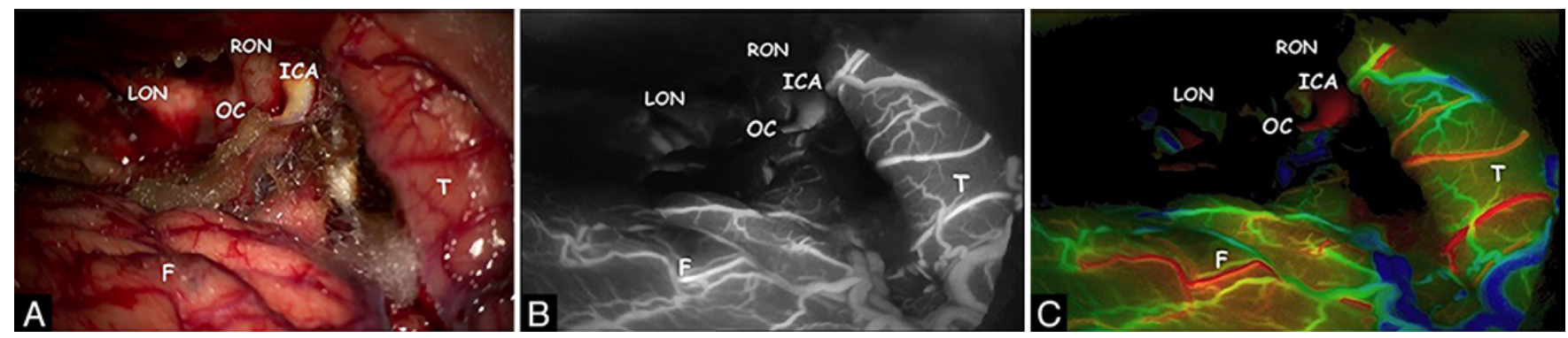

FIG. 6. Planum sphenoidalis meningioma infiltrating the optic chiasm $(O C)$, with bilateral encasement of $I C A$, $A_{1}$, and their perforators, and compression of the left and right optic nerves (LON, RON). Even if at white-light microscopy examination (A) the optic chiasm appeared intact, the postresection ICG-VA (B) with FLOW 800 analysis (C) showed chiasmal hypoperfusion, whereas ICA and parenchyma were normally perfused. The patient suffered from severe vision problems due to optic chiasm damage, although no other neurological disturbances related to brain damage were evident in the postoperative period. $\mathrm{F}=$ frontal; $\mathrm{T}=$ temporal. 

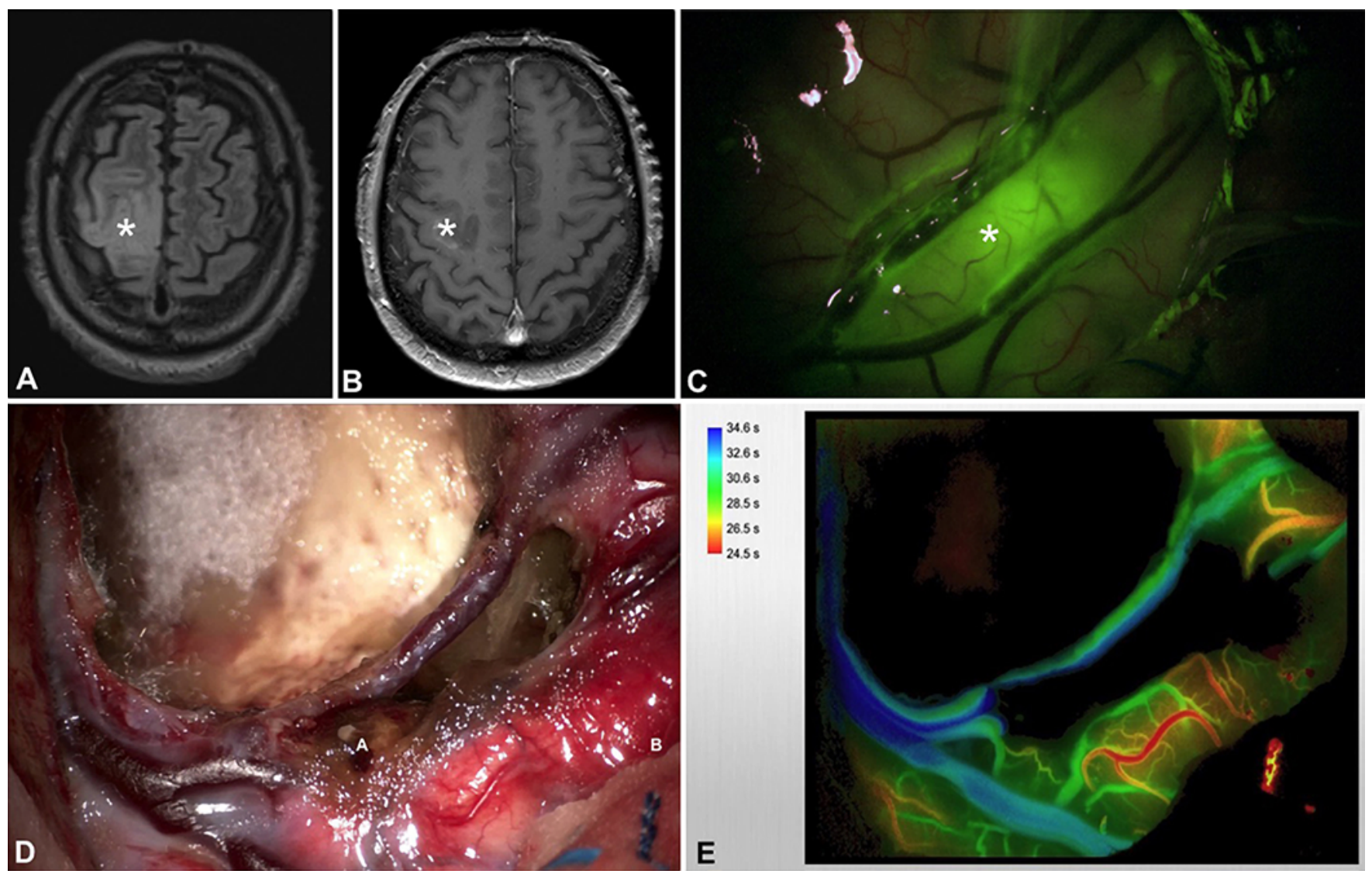

FIG. 7. Right frontal oligodendroglioma with anaplastic component (asterisks in A-C). The tumor was removed using the fluorescein-guided technique and appeared brightly fluorescent under filter activation (C). After tumor removal (D), ICG-VA was performed and a surgically manipulated vein was demonstrated to be patent, with anterograde flow toward the SSS in the map of delay times $(\mathbf{E})$.

meningiomas), 4 of which were further analyzed after previous studies performed for other indications (see previous paragraphs), ICG-VA with FLOW 800 analysis was performed postoperatively to directly assess brain or spinal cord perfusion even without artery manipulation during surgical maneuvers. In 5 parasagittal meningiomas, the same ICG-VA study and, more precisely, the use of maps of delay times and flow curve analysis based on FLOW 800 software allowed us to evaluate the patency and flow characteristics of the veins manipulated during resection. No flow perturbation could be found in this subgroup of patients.

In 8 cases ( 1 frontomesial anaplastic oligodendroglioma, 1 central neurocytoma, 5 meningiomas, 1 tumor of pineal gland region) ICG-VA with FLOW 800 analysis was performed to evaluate only venous patency and flow after tumor resection, in veins not studied in the preoperative survey (in 5 studies to assess cortical veins draining to the SSS, in 1 study to assess the patency of internal cerebral veins, in 1 study to assess direction of flow in superficial sylvian veins after sacrifice of a draining vein to the spheno-petrosal sinus, and in 1 study to assess the patency of precerebellar veins afferent to the vein of Galen). In all but 2 cases the veins were patent and with anterograde flow toward the sinuses (Fig. 7); in 1 case the portion of the vein directly connected to the SSS was occluded but with collateral retrograde flow, as evident on the map of delay times; in 1 case the sylvian vein drained in a retrograde way toward the Labbé system.

In 1 case (1 meningioma), ICG-VA was performed to confirm the persistence of anastomotic circulation in veins after their sacrifice.

In 5 cases ( 3 GBMs, 1 metastasis, and 1 hemangioblastoma) ICG-VA with FLOW 800 analysis was performed postoperatively to study flow in veins that presented with an AVF before tumor resection. In all cases the postoperative survey with FLOW 800 analysis confirmed the normalization of venous flow after tumor resection (Figs. 3 and 4).

\section{Discussion}

In 2011, we were the first to propose the use of classic ICG-VA during the resection of CNS tumors, based on a retrospective series of patients who were treated with resection in the period 2006-2008. ${ }^{11}$ However, at that time, the technique for semiquantitative analysis of blood flow integrated in the surgical microscope was not available yet. With FLOW 800 software, a semiquantitative analysis of blood flow in the brain area exposed by the craniotomy could be performed. In particular, the algorithm integrated in the surgical microscope is able to reconstruct maps of 
gray intensity based on the average AIs of infrared fluorescence detected by the microscope camera, and maps of delay time based on the time needed to reach $50 \%$ of the maximal fluorescence intensity. In addition, it is possible to study in multiple ROIs the curves of fluorescence detected by the camera over the time of ICG-VA registration.

Kamp et al. were the first authors to explore the use of microscope-integrated quantitative analysis of ICG-VA for blood flow assessment. ${ }^{24}$ These authors studied 30 patients with only vascular pathologies, and they were the first to hypothesize the possibility of using the different parameters derived from FLOW 800 analysis in clinical applications. In particular they showed that the maps of delay time appeared to be of great value, in giving an immediate representation of blood flow perturbation in cortical areas, either as an effect of the disease itself, or related to iatrogenic damage to arteries manipulated in the surgical approach. Despite several applications of ICG-VA that have been described so far, $8,9,16,23,25,28,30-32,34,35,39,40$ it is remarkable to note the paucity of articles reporting its use, along with quantitative analysis to explore cerebral perfusion in the intraoperative setting. ${ }^{38,41}$ Even less interest could be found in the context of surgery for brain tumors, where only cerebral and spinal hemangioblastomas have been studied. , $20,37^{2}$

In 2009 we acquired the technology to use the FLOW 800 algorithm for flow analysis in both vascular and tumor cases. We identified 73 tumor cases in which ICG-VA was used, and in which both surgical reports and videos were available for retrospective analysis, in patients treated between May 2011 and December 2017. Thus, only approximately $5 \%$ of the entire tumor population that was surgically treated in the same period was selected for ICG-VA. Our population consisted of a heterogeneous group of cerebral and spinal tumors, represented mainly by meningiomas and high-grade gliomas. The most frequent application for ICG-VA was in a postresection survey, in particular to assess arterial and venous patency and brain perfusion after tumor resection. In $87.5 \%$ of the cases the postresection survey confirmed arterial and venous patency and the absence of local hypoperfusion, thus providing assurances on the low risk of postoperative complications related to vessel manipulation. On the contrary, in the remaining $12.5 \%$ of the cases the postoperative survey showed local hypoperfusion directly related to vessel manipulation. In 2 of these cases, the intraoperative recognition of this complication allowed the surgeons to perform surgical maneuvers to reverse the local hypoperfusion and reduce the risk of postoperative complications. ${ }^{3}$ Even though a similar experience could be attained also with classic ICG-VA, as already reported in our previous paper and in a similar study by other authors, ${ }^{11,27}$ the use of blood flow analysis by FLOW 800 software allowed us to better interpret the brain perfusion, particularly with the maps of delay times, as indicated also in vascular experience (Figs. 3, 4, 6, and 7). ${ }^{24}$

The preoperative survey, performed in 27 cases, was used to identify both specific tumor-related pathophysiological vascular changes and nonspecific mass-effect changes. This was particularly important in GBM and hemangioblastomas, in which the superficial vascular pattern and the presence of AVFs could be clearly shown (Figs. 3 and 4). The possibility of identifying the feeding arteries and the tumor nodule in hemangioblastomas was reported also by Hojo et al. in 2014, ${ }^{20}$ but mainly with classic ICGVA. However, we also believe that for this application the use of semiquantitative flow analysis improved and simplified the interpretation of videoangiographic data. Furthermore, preoperative survey and long recording time allowed us to concentrate our attention on the venous flow and, when needed, to perform a temporary clipping test to decide whether or not the vein could be sacrificed to enlarge the surgical corridor or completely resect the tumor, as shown by our group in a preliminary experience in 8 cases. ${ }^{14}$ We never performed an arterial clipping test for arterial sacrifice, as proposed by Kim and Cohen-Gadol in $2013,{ }^{26}$ but we also believe that this application could be expanded in the future. An innovation compared to previous experiences was also the use of ICG-VA in spinal tumors as a way to help in posterior median sulcus identification when the tumor was not abutting spinal cord survey, and when, due to edema and congestion, the normal anatomy was distorted and the sulcus was difficult to find with white-light illumination. We were also able to show, in very select cases (only 6 cases in our series), that an extradural survey was feasible, and that allowed identification of both dural sinus and cortical vein courses, thus helping in dural opening, as already shown by Nussbaum et al. ${ }^{29}$

We are aware of the fact that our study is only a retrospective evaluation of the different application of ICG-VA with FLOW 800 analysis, in a limited series of patients with CNS tumors, based on the specific experience of our group. In addition, we did not perform any comparison with other techniques of blood flow monitoring, and thus we could not provide any measurement about sensitivity and specificity. However, we believe that the results of this paper underline the concept that the surgery of brain tumors is also a surgery of brain vessels, and that understanding the pathophysiological changes of the brain circulation that are directly related to the tumor presence and sometimes to the surgical manipulation, as derived by flow analysis provided by ICG-VA, could be associated with better surgical results in selected cases.

\section{Conclusions}

We found ICG-VA with FLOW 800 to be a useful method to monitor blood flow in the exposed vessels and parenchyma during microsurgical removal of CNS tumors in selected cases. In particular a preresection survey provides useful information about pathophysiological changes of brain vasculature related to the tumor and individuates helpful landmarks for the surgical approach, and the postresection survey helps to prevent potential complications associated with the resection (such as local hypoperfusion or venous infarction). Future studies with a larger tumor population should be performed to confirm all the potential advantages of this technique in the surgical management of CNS tumors.

\section{References}

1. Acerbi F, Broggi M, Schebesch KM, Höhne J, Cavallo C, De Laurentis C, et al: Fluorescein-guided surgery for resection 
of high-grade gliomas: a multicentric prospective phase II study (FLUOGLIO). Clin Cancer Res 24:52-61, 2018

2. Acerbi F, Cavallo C, Broggi M, Cordella R, Anghileri E, Eoli M, et al: Fluorescein-guided surgery for malignant gliomas: a review. Neurosurg Rev 37:547-557, 2014

3. Acerbi F, Cavallo C, Ferroli P: Intraoperative assessment of blood flow with quantitative indocyanine green videoangiography: the role for diagnosis of regional cerebral hypoperfusion. Neurosurgery 78:E310-E312, 2016 (Letter)

4. Acerbi F, Cavallo C, Schebesch KM, Akçakaya MO, de Laurentis C, Hamamcioglu MK, et al: Fluorescein-guided resection of intramedullary spinal cord tumors: results from a preliminary, multicentric, retrospective study. World Neurosurg 108:603-609, 2017

5. Acerbi F, Restelli F, Broggi M, Schiariti M, Ferroli P: Feasibility of simultaneous sodium fluorescein and indocyanine green injection in neurosurgical procedures. Clin Neurol Neurosurg 146:123-129, 2016

6. Benedetto N, Aquila F, Vannozzi R: Use of near-infrared indocyanine videoangiography and Flow 800 in the resectioning of a spinal cord haemangioblastoma. Br J Neurosurg 27:847-849, 2013

7. d'Avella E, Volpin F, Manara R, Scienza R, Della Puppa A: Indocyanine green videoangiography (ICGV)-guided surgery of parasagittal meningiomas occluding the superior sagittal sinus (SSS). Acta Neurochir (Wien) 155:415-420, 2013

8. Dashti R, Laakso A, Niemelä M, Porras M, Hernesniemi J: Microscope-integrated near-infrared indocyanine green videoangiography during surgery of intracranial aneurysms: the Helsinki experience. Surg Neurol 71:543-550, 2009

9. de Oliveira JG, Beck J, Seifert V, Teixeira MJ, Raabe A: Assessment of flow in perforating arteries during intracranial aneurysm surgery using intraoperative near-infrared indocyanine green videoangiography. Neurosurgery 61 (3 Suppl):63-73, 2007

10. Endo T, Aizawa-Kohama M, Nagamatsu K, Murakami K, Takahashi A, Tominaga T: Use of microscope-integrated near-infrared indocyanine green videoangiography in the surgical treatment of intramedullary cavernous malformations: report of 8 cases. J Neurosurg Spine 18:443-449, 2013

11. Ferroli P, Acerbi F, Albanese E, Tringali G, Broggi M, Franzini A, et al: Application of intraoperative indocyanine green angiography for CNS tumors: results on the first 100 cases. Acta Neurochir Suppl 109:251-257, 2011

12. Ferroli P, Acerbi F, Broggi M, Broggi G: Arteriovenous micromalformation of the trigeminal root: intraoperative diagnosis with indocyanine green videoangiography: case report Neurosurgery 67 (3 Suppl Operative):E309-E310, 2010

13. Ferroli P, Acerbi F, Broggi M, Broggi G: The role of indocyanine green videoangiography (ICGV) in surgery of parasagittal meningiomas. Acta Neurochir (Wien) 155:1035, 2013

14. Ferroli P, Acerbi F, Tringali G, Albanese E, Broggi M, Franzini A, et al: Venous sacrifice in neurosurgery: new insights from venous indocyanine green videoangiography. J Neurosurg 115:18-23, 2011

15. Ferroli P, Nakaji P, Acerbi F, Albanese E, Broggi G: Indocyanine green (ICG) temporary clipping test to assess collateral circulation before venous sacrifice. World Neurosurg 75:122-125, 2011

16. Ferroli P, Tringali G, Albanese E, Broggi G: Developmental venous anomaly of petrous veins: intraoperative findings and indocyanine green video angiographic study. Neurosurgery 62 (5 Suppl 2):ONS418-ONS422, 2008

17. Hao S, Li D, Ma G, Yang J, Wang G: Application of intraoperative indocyanine green videoangiography for resection of spinal cord hemangioblastoma: advantages and limitations. J Clin Neurosci 20:1269-1275, 2013

18. Hettige S, Walsh D: Indocyanine green video-angiography as an aid to surgical treatment of spinal dural arteriovenous fistulae. Acta Neurochir (Wien) 152:533-536, 2010

19. Hide T, Yano S, Shinojima N, Kuratsu J: Usefulness of the indocyanine green fluorescence endoscope in endonasal transsphenoidal surgery. J Neurosurg 122:1185-1192, 2015

20. Hojo M, Arakawa Y, Funaki T, Yoshida K, Kikuchi T, Takagi Y, et al: Usefulness of tumor blood flow imaging by intraoperative indocyanine green videoangiography in hemangioblastoma surgery. World Neurosurg 82:e495-e501, 2014

21. Hope-Ross M, Yannuzzi LA, Gragoudas ES, Guyer DR, Slakter JS, Sorenson JA, et al: Adverse reactions due to indocyanine green. Ophthalmology 101:529-533, 1994

22. Hwang SW, Malek AM, Schapiro R, Wu JK: Intraoperative use of indocyanine green fluorescence videography for resection of a spinal cord hemangioblastoma. Neurosurgery 67 (3 Suppl Operative):ons300-ons303, 2010

23. Imizu S, Kato Y, Sangli A, Oguri D, Sano H: Assessment of incomplete clipping of aneurysms intraoperatively by a nearinfrared indocyanine green-video angiography (Niicg-Va) integrated microscope. Minim Invasive Neurosurg 51:199_ 203, 2008

24. Kamp MA, Slotty P, Turowski B, Etminan N, Steiger HJ, Hänggi D, et al: Microscope-integrated quantitative analysis of intraoperative indocyanine green fluorescence angiography for blood flow assessment: first experience in 30 patients. Neurosurgery 70 (1 Suppl Operative):65-74, 2012

25. Killory BD, Nakaji P, Gonzales LF, Ponce FA, Wait SD, Spetzler RF: Prospective evaluation of surgical microscopeintegrated intraoperative near-infrared indocyanine green angiography during cerebral arteriovenous malformation surgery. Neurosurgery 65:456-462, 2009

26. Kim DL, Cohen-Gadol AA: Indocyanine-green videoangiogram to assess collateral circulation before arterial sacrifice for management of complex vascular and neoplastic lesions: technical note. World Neurosurg 79:404.e1-404.e6, 2013

27. Kim EH, Cho JM, Chang JH, Kim SH, Lee KS: Application of intraoperative indocyanine green videoangiography to brain tumor surgery. Acta Neurochir (Wien) 153:14871495,2011

28. Li J, Lan Z, He M, You C: Assessment of microscope-integrated indocyanine green angiography during intracranial aneurysm surgery: a retrospective study of 120 patients. Neurol India 57:453-459, 2009

29. Nussbaum ES, Defillo A, Nussbaum L: The use of indocyanine green videoangiography to optimize the dural opening for intracranial parasagittal lesions. Neurosurgery 70 (1 Suppl Operative):61-64, 2012

30. Raabe A, Beck J, Gerlach R, Zimmermann M, Seifert V: Near-infrared indocyanine green video angiography: a new method for intraoperative assessment of vascular flow. Neurosurgery 52:132-139, 2003

31. Raabe A, Beck J, Seifert V: Technique and image quality of intraoperative indocyanine green angiography during aneurysm surgery using surgical microscope integrated near-infrared video technology. Zentralbl Neurochir 66:1-8, 2005

32. Raabe A, Nakaji P, Beck J, Kim LJ, Hsu FP, Kamerman JD, et al: Prospective evaluation of surgical microscopeintegrated intraoperative near-infrared indocyanine green videoangiography during aneurysm surgery. J Neurosurg 103:982-989, 2005

33. Schebesch KM, Hoehne J, Hohenberger C, Acerbi F, Broggi M, Proescholdt M, et al: Fluorescein sodium-guided surgery in cerebral lymphoma. Clin Neurol Neurosurg 139:125128,2015

34. Takagi Y, Kikuta K, Nozaki K, Sawamura K, Hashimoto $\mathrm{N}$ : Detection of a residual nidus by surgical microscopeintegrated intraoperative near-infrared indocyanine green videoangiography in a child with a cerebral arteriovenous malformation. J Neurosurg 107 (5 Suppl):416-418, 2007 
35. Takagi Y, Sawamura K, Hashimoto N, Miyamoto S: Evaluation of serial intraoperative surgical microscope-integrated intraoperative near-infrared indocyanine green videoangiography in patients with cerebral arteriovenous malformations. Neurosurgery 70 (1 Suppl Operative):34-43, 2012

36. Takami T, Naito K, Yamagata T, Shimokawa N, Ohata K: Benefits and limitations of indocyanine green fluorescent image-guided surgery for spinal intramedullary tumors. Oper Neurosurg (Hagerstown) 13:746-754, 2017

37. Takeshima Y, Tanaka Y, Hironaka Y, Shida Y, Nakase H: Visualization of vascular structure of spinal hemangioblastoma using intraoperative indocyanine green videoangiography and temporary feeder occlusion. Eur Spine J 24 (Suppl 4):S585-S589, 2015

38. Uchino H, Kazumata K, Ito M, Nakayama N, Kuroda S, Houkin K: Intraoperative assessment of cortical perfusion by indocyanine green videoangiography in surgical revascularization for moyamoya disease. Acta Neurochir (Wien) 156: $1753-1760,2014$

39. Woitzik J, Horn P, Vajkoczy P, Schmiedek P: Intraoperative control of extracranial-intracranial bypass patency by nearinfrared indocyanine green videoangiography. J Neurosurg 102:692-698, 2005

40. Xu BN, Sun ZH, Romani R, Jiang JL, Wu C, Zhou DB, et al: Microsurgical management of large and giant paraclinoid aneurysms. World Neurosurg 73:137-146, e17, e19, 2010

41. Ye X, Liu XJ, Ma L, Liu LT, Wang WL, Wang S, et al: Clinical values of intraoperative indocyanine green fluorescence video angiography with Flow 800 software in cerebrovascular surgery. Chin Med J (Engl) 126:4232-4237, 2013

\section{Disclosures}

Dr. Acerbi received speaker's fees from Carl Zeiss Meditec for lectures at International Congresses.

\section{Author Contributions}

Conception and design: Acerbi, Vetrano, Ferroli. Acquisition of data: Acerbi, Vetrano, Sattin, de Laurentis, Bosio, Broggi, Schiariti. Analysis and interpretation of data: all authors. Drafting the article: Acerbi, Vetrano, Sattin, de Laurentis, Schiariti, Ferroli. Critically revising the article: Acerbi, Vetrano, Rossini, Broggi, Ferroli. Reviewed submitted version of manuscript: Acerbi, Vetrano. Approved the final version of the manuscript on behalf of all authors: Acerbi. Study supervision: Acerbi, Ferroli.

\section{Supplemental Information Videos \\ Video Abstract. https://vimeo.com/269639196.}

\section{Correspondence}

Francesco Acerbi: Fondazione IRCCS "Istituto Neurologico Carlo Besta,” Milan, Italy. francesco.acerbi@istituto-besta.it. 